\title{
Mean Corpuscular Volume is Equivalent to $\gamma$-GTP as an Indicator of Alcohol Intake
}

\author{
Tsutomu FuKUMOTo ${ }^{1}$; Takashi WADA ${ }^{1}$; and Atsuko KURISU ${ }^{1}$ \\ ${ }^{1}$ Health Science Center, The Jikei University
}

\section{ABSTRACT}

Objective Although Gamma-glutamyl transpeptidase ( $\boldsymbol{\gamma}$ GTP) is known as an alcohol indicator, it does not parallel alcohol intake, especially in women. In addition, mean corpuscular volume (MCV) is not widely used as an indicator of alcohol intake. The purpose of this article is to determine the efficacy of MCV as an indicator of alcohol intake, and the cutoff value of $\mathrm{MCV}$ in the population consuming appropriate amount of alcohol.

Metbod (1) Total of 6,989 subjects aged 30 to 69 years were recruited during our health check-up, and MCV and $\gamma$-GTP were compared between different Ethanol groups. (2) The cutoff values for MCV and $\gamma$-GTP corresponding to the appropriate Ethanol (150 $\mathrm{g}$ in men, and $100 \mathrm{~g}$ in women) were calculated using receiver operating characteristic curves. Ethanol is alcohol intake per week expressed in gram of ethanol.

Results (1) MCV elevated with the increase of Ethanol in both sex. $\gamma$-GTP in men increased parallel to Ethanol, but not in women. (2) The cut-off values for MCV and $\gamma$-GTP corresponding to the appropriate Ethanol were $92 \mathrm{fL}$ and $40 \mathrm{IU} / \mathrm{L}$ in men, and $90 \mathrm{fL}$ and $17 \mathrm{IU} / \mathrm{L}$ in women, respectively.

Conclusion (1) MCV is an indicator of alcohol intake in both sex, while $\gamma$-GTP is an indicator only in men. (2) MCV is equivalent to $\gamma$-GTP in terms of indicating the appropriate alcohol intake. Overall, MCV is equivalent to $\gamma$-GTP as an alcohol indicator of appropriate alcohol intake.

Key Words Cut-off Value, Appropriate Alcohol Intake

\section{INTRODUCTION}

Excessive alcohol intake is a risk factor of various diseases including liver dysfunction, hypertension, gout, and cerebral hemorrhage as well as alcohol dependence, a mental disorder. Gamma-glutamyl transpeptidase ( $\gamma$-GTP) has been mainly used as a subjective indicator of alcohol intake. However, the elevation of $\gamma$-GTP is not always proportional to the alcohol intake, especially in women.

As an alternative, we focused on the mean corpuscular volume (MCV), a screening examination widely used in the health check-up, and determined its efficacy as a subjective indicator of the alcohol intake in comparison with $\gamma$-GTP. The reference range of MCV has been reported to be 80-100 fL. ${ }^{[1]}$ A study showed that MCV was affected by drinking even in this reference range. ${ }^{[2]}$ Therefore, we calculated the cut-off value of MCV corresponding to the appropriate alco-

Received: December 10, 2003

${ }^{1}$ Address; Health Science Center, The Jikei University, 3-25-8, Nishi-Shimbashi, Minato-ku, Tokyo, 105-8461 JAPAN. TEL: 813-3433-1111 (ex5281), FAX: 81-3-5472-2584, E-mail: hol intake.

\section{SUBJECTS}

Subjects were 30 to 69 year-old Japanese, who received health check-up at the Health Science Center in the Jikei University between January and December, 2000. Since MCV is dependent on iron or vitamin $\mathrm{B}_{12}$ absorption, ${ }^{[3], ~[4]}$ subjects with a history of gastrectomy, or with serum iron levels lower than the minimum reference value $(60-165 \mu \mathrm{g} / \mathrm{dL}$ for men, and $40-145 \mu \mathrm{g} / \mathrm{dL}$ for women $)^{[3]}$ were excluded. Subjects with positive hepatitis $B$ antigen or positive hepatitis $C$ antibody were also excluded, because $\gamma$-GTP is influenced by hepatitis B and C. ${ }^{[5]}$ Total of 6,989 subjects participated, of which 5,391 were men (mean age $49.8 \pm 10.8$ years) and 1,598 were women (mean age $47.0 \pm 11.2$ ).

\section{METHODS}

\section{(a) Stratification by the alcohol intake}

Questionnaires were used for surveying Age, Days, Years and Ethanol.

Age is subjects' age. Days is frequency of drinking per week expressed in days. Years is duration of drinking expressed in years. Ethanol is alcohol intake per week expressed in grams of ethanol. ${ }^{[6]}$ The National Nutrition Survey in Japan revealed the incidence of drinking more than ethanol $22 \mathrm{~g}$ per day was $2.3 \%$ in women, about one-tenth of men $(25 \%){ }^{[7]}$ Clinical epidemiology has shown that women develop cirrhosis with smaller amount of alcohol and in shorter period than men. ${ }^{[8]}$ Because sex difference in alcohol intake and its effect is apparent from these studies, we divided subjects into five groups by sex and Ethanol as follows;

Men: Group 1, no drinking ( $n=830$, mean age 49.8 \pm 10.4$)$; Group 2, Ethanol<150 g $(n=2,132,47.7 \pm 10.4)$; Group 3, Ethanol $\geqq 150$ and $<300 \mathrm{~g}(n=1,464,50.6 \pm 9.3)$; Group 4, Ethanol $\geqq 300$ and $<450 \mathrm{~g}(n=654,51.0 \pm 8.8)$; Group 5, Ethanol $\geqq 450 \mathrm{~g}(n=311,50.8 \pm 8.9)$.

Women: Group 1, no drinking ( $n=886,48.0 \pm 10.2)$; Group 2 , Ethanol $<50 \mathrm{~g}(n=317,45.0 \pm 9.8)$; Group 3, Ethanol $\geqq 50$ and $<100 \mathrm{~g}(n=139,44.1 \pm 9.0)$; Group 4, Ethanol $\geqq 100$ and $<150 \mathrm{~g}(n=98,45.6 \pm 9.0)$; Group 5, Ethanol $\geqq 150 \mathrm{~g}(n=158$, 46.2 \pm 8.8$)$.

MCV was measured by flow cytometory using XE2100 automated hematology analyzer (Sysmex, Hyogo). $\gamma$-GTP was measured by enzyme method using 7,600 clinical biochemistry analyzer (HITACHI, Tokyo). Reagent was $\gamma$-GTP 
(3C)-SL (Sysmex, Hyogo). Reference value was 0-50 IU/L for men, and 0-30 IU/L for women. ${ }^{[5]}$

The results were expressed as mean \pm SD (standard deviation). The mean values of MCV and $\gamma$-GTP were compared between groups using the analysis of variance (Tukey-Kremer method) and the $p<0.05$ was considered significant. Subsequently, regression analysis (Stepwise method) was performed, where MCV and $\gamma$-GTP were dependent variables and Age, Days, Years, and Ethanol were independent variables. The value of $\mathrm{F} \geqq 4$ was considered significant. Stat View 5.0 software (SAS Institute Inc., Cary, USA) was used for the statistical analysis. Eight hundred and two men and 797 women were excluded from the regression analysis, since they did not provide Years in the questionnaires.

\section{(b)Age group}

Both men and women were divided into four groups by their age, because MCV is influenced by age. ${ }^{[9]}$

Men: 30-39 $(n=1,199), 40-49(n=1,332), 50-59(n=1,878)$, 60-69 $(n=982)$

Women: $30-39(n=462), 40-49(n=486), 50-59(n=463)$, 60-69 $(n=187)$

MCV and $\gamma$-GTP were compared between age groups by the analysis of variance as described above. (c) MCV and $\boldsymbol{\gamma}$-GTP values corresponding to the appropriate alcohol intake

According to the National Health Promotion in the 21st Century, Health Japan 21, the recommended alcohol intake is less than or equal to ethanol $22 \mathrm{~g}$ per day. ${ }^{[10]}$ We estimated the appropriate Ethanol is less than $150 \mathrm{~g}$ for men and less than $100 \mathrm{~g}$ for women, because the effects of alcohol on health are greater in women than in men. ${ }^{[8]}$ We generated receiver operating characteristic curve (ROC curve) for MCV or $\gamma$-GTP being a variable, and determined the cut-off value corresponding to the appropriate Ethanol of $150 \mathrm{~g}$ in men and $100 \mathrm{~g}$ in women. The effective range was set to 50.1-99.9\%. SPSS10.0 software (SPSS Inc., Chicago, USA) was used for the analysis. The sensitivity and specificity of cut-off values for MCV and $\gamma$-GTP were determined in men and in women.

\section{RESULTS}

\section{(a) Stratification by Ethanol}

MCV elevated both in men and women as Ethanol increased (Figs. 1 and 2). $\gamma$-GTP elevated as Ethanol increased in men. In women, however, $\gamma$-GTP did not significantly vary between Groups 1-4 (Figs. 3 and 4).

\section{fL}
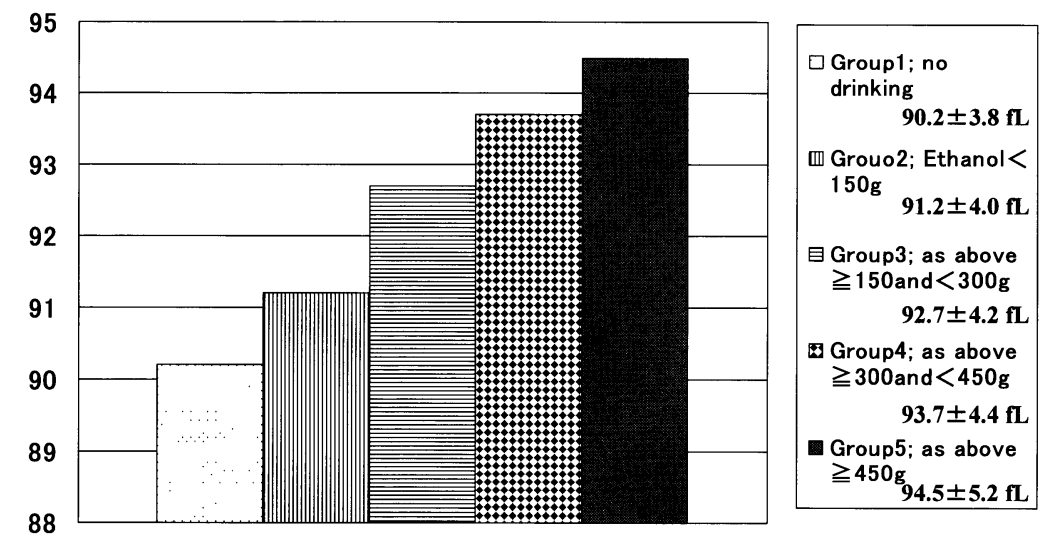

$\mathrm{MCV}=$ mean corpuscular volume Ethanol=alcohol intake per week expressed in gram of ethanol

Fig. 1 MCV value by Ethanol in men.
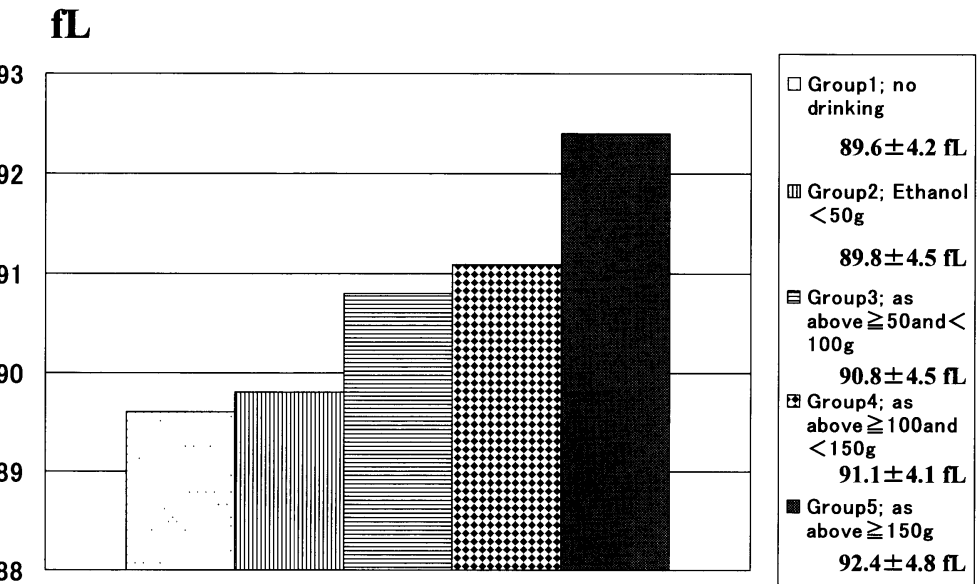

Fig. 2 MCV value by Ethanol in women. 
IU/L

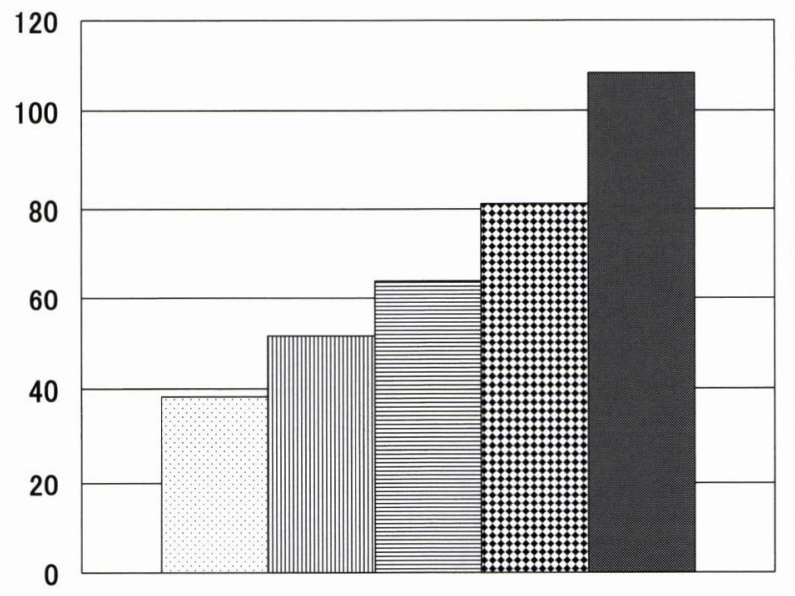

$\bigoplus$ Group 1 ; no drinking $38.4 \pm 35.9 \mathrm{IU} /$

四 Group2; Ethanol< $150 \mathrm{~g}$

$51.5 \pm 61.8 \mathrm{IU} / \mathrm{L}$

目 Group3; as above $\geqq$ 150 and $<300 \mathrm{~g}$

$64.0 \pm 62.9 \mathrm{IU} / \mathrm{L}$

Group4; as above $\geqq$

300 and $<450 \mathrm{~g}$

$81.0 \pm 84.6 \mathrm{IU} / \mathrm{L}$

Group5; as above $\geqq$

$450 \mathrm{~g}_{108.4 \pm 109.5 \mathrm{IU} / \mathrm{L}}$

$\gamma-\mathrm{GTP}=$ gamma-glutamyl transpeptidase

Fig. $3 \gamma$-GTP value by Ethanol in men.

IU/L
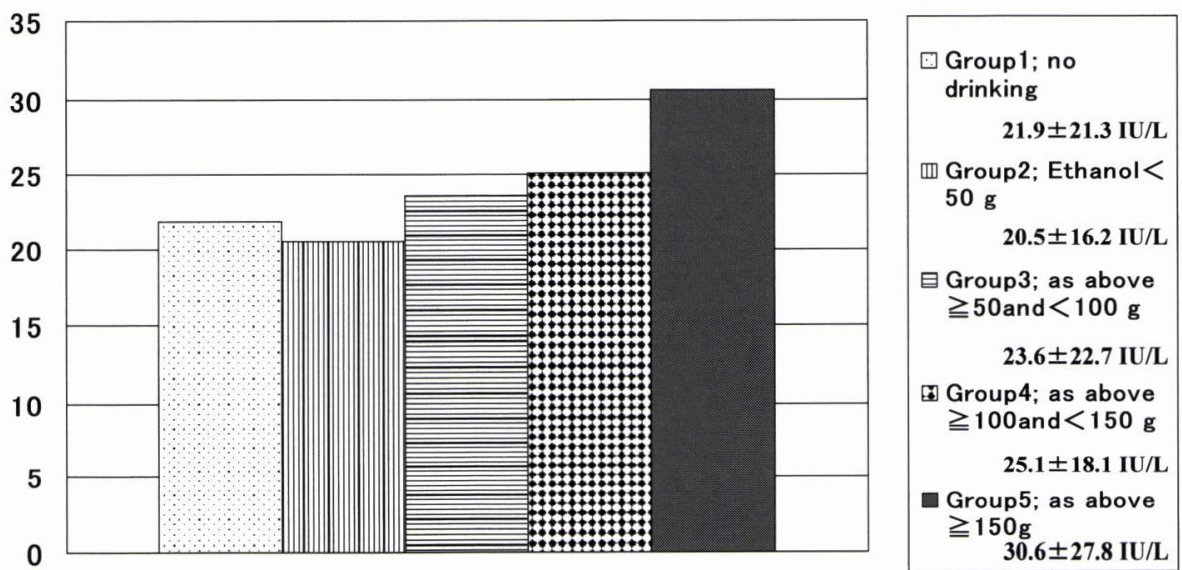

Fig. $4 \gamma$-GTP value by Ethanol in women.

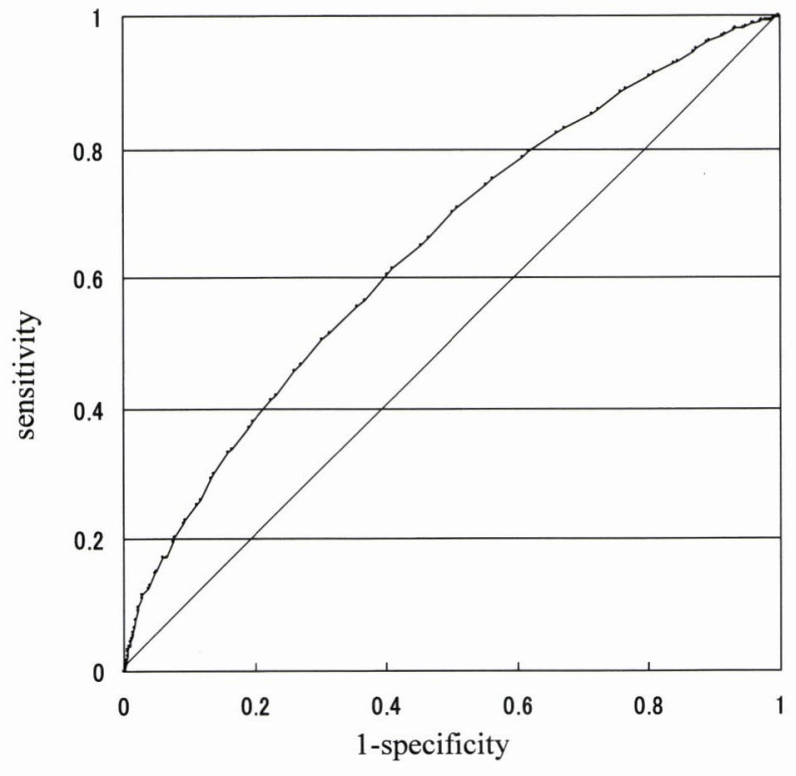

ROC curve $=$ receiver operating characteristic curve

Fig. 5 ROC curve of MCV in men.

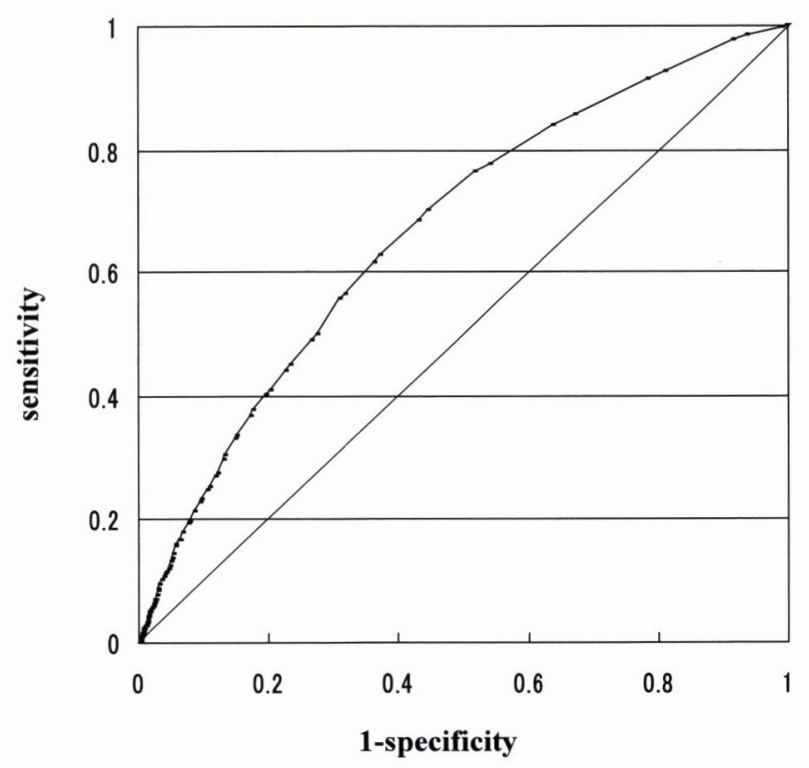

Fig. 6 ROC curve of $\gamma$-GTP in men. 


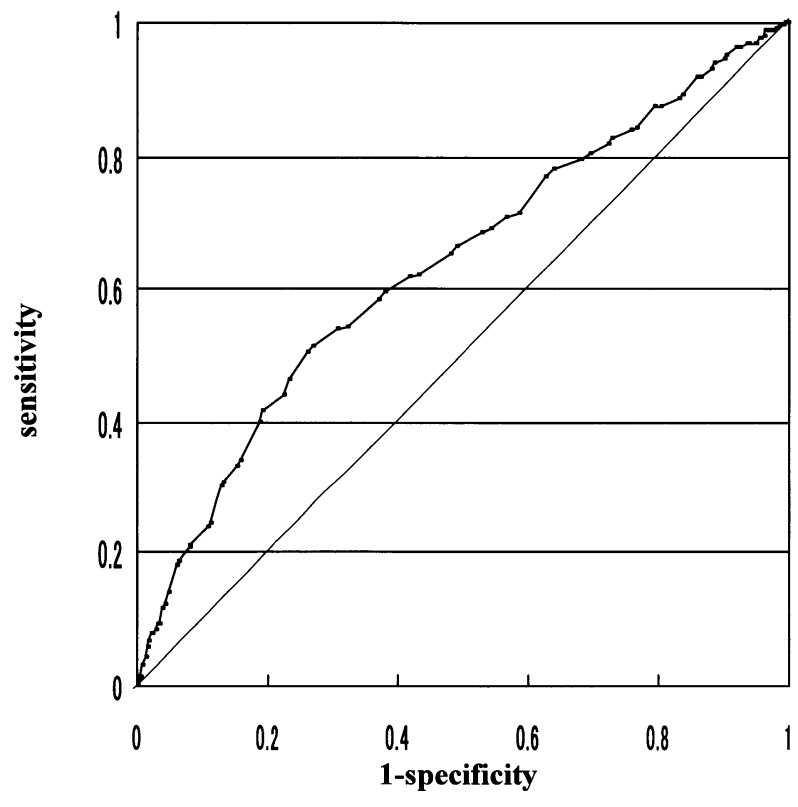

Fig. 7 ROC curve of MCV in women.

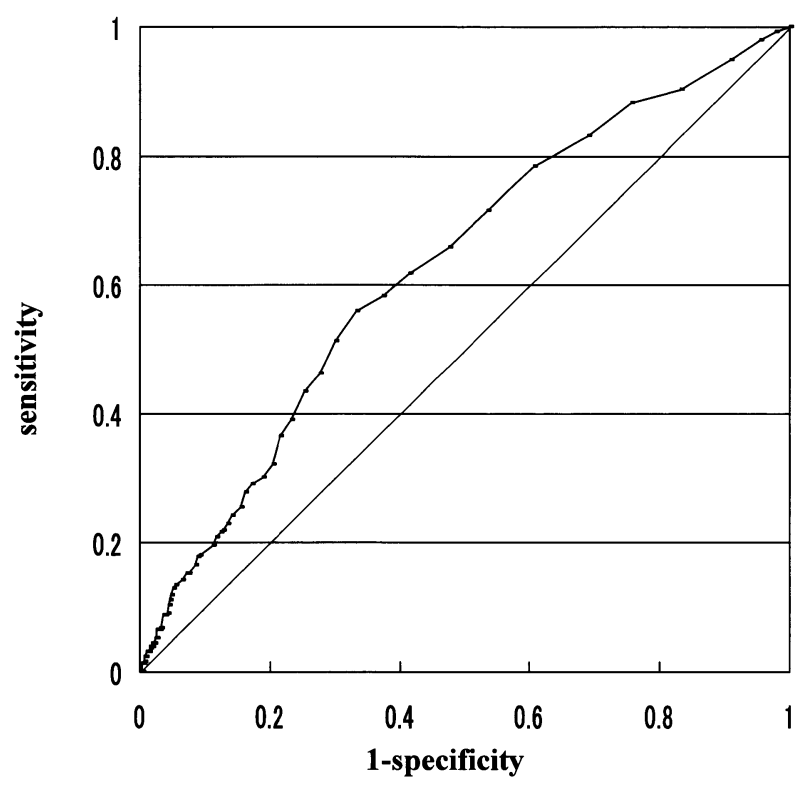

Fig. 8 ROC curve of $\gamma$-GTP in women.

MCV in men was significantly correlated to Age $(\mathrm{F}=166.9)$, Ethanol $(\mathrm{F}=115.9)$, Days $(\mathrm{F}=73.6)$, and Years $(\mathrm{F}=13.8) . \quad \gamma$-GTP in men was correlated to Ethanol $(\mathrm{F}=219.4)$ and Years $(\mathrm{F}=10.9)$. MCV in women was correlated to Ethanol $(\mathrm{F}=52.5)$ and Years $(\mathrm{F}=16.3) . \quad \gamma$-GTP in women was correlated to Age $(\mathrm{F}=42.4)$ and Ethanol $(\mathrm{F}=11.9)$.

\section{(b) Age group}

MCV elevated as age got older in both sex. $\gamma$-GTP in both sex increased from $30 \mathrm{~s}$ to $50 \mathrm{~s}$, and then decreased from 50 s to $60 \mathrm{~s}$.

(c) MCV and $\gamma$-GTP values corresponding to the appropriate Ethanol

In men the cut-off value for MCV was $92 \mathrm{fL}$ (sensitivity $60.5 \%$, specificity $59.8 \%$, area 64.6\%) (Fig. 5) and the value for $\gamma$-GTP was $40 \mathrm{IU} / \mathrm{L}(62.9 \%, 62.9 \%, 67.1 \%)$ (Fig. 6). In women the cut-off value for MCV was $90 \mathrm{fL}(64.1 \%, 53.3 \%$, $63.6 \%$ ) (Fig. 7) and the value for $\gamma$-GTP was $17 \mathrm{IU} / \mathrm{L}(61.7 \%$, $58.6 \%, 62.9 \%$ ) (Fig. 8). MCV was equivalent to $\gamma$-GTP as an indicator of appropriate alcohol intake.

\section{DISCUSSION}

The indicators of alcohol intake include inquiry methods and blood examination. The inquiry methods including Michigan alcoholism screening test (MAST) are mainly used for the early detection of alcohol dependence. ${ }^{[11]}$ It is known that the amount of alcohol described by alcoholics is not reliable, although their description of frequency of drinking is reliable to some extent. Therefore there aren't suitable for applying to our subjects. We used usual inquiry method to estimate alcohol amount in this study, because our subjects were mainly company workers and their family members, and included few excessive drinkers who required treatment.

Blood examination related to drinking includes IgA, cytokines, markers of liver fibrosis, carbohydrate deficient transferin (CDT), and $\gamma$-GTP. Among these tests, $\gamma$-GTP is a routine laboratory examination. Alcohol has enzyme-inducing effect and increases $\gamma$-GTP contents in the liver. This increase is reflected to the elevation of $\gamma$-GTP preceding the rise in GOT and GPT, and abstinence decreases $\gamma$-GTP. The drawback of $\gamma$-GTP as an indicator of alcohol intake is that its rise is not proportional to alcohol amount; small amount of alcohol may cause elevation of $\gamma$-GTP and large amount of alcohol may not. Although CDT is a valuable indicator that elevates with alcohol abuse, ${ }^{[12]}$ it is not a routine examination and is not used in regular health check-up.

MCV is one of the most basic laboratory examinations and used for differentiation of various types of anemia. Although increased MCV suggests megaloblastic anemia or pernicious anemia, alcohol intake may be the cause of elevated MCV without anemia. ${ }^{[13]-[15]}$ The mechanism of increased MCV by drinking is not clear. One possible explanation is that alcohol intake causes changes in the lipid composition of red blood cell membrane in vitro test, and results in the increased MCV. ${ }^{[16]}$

In this study, MCV showed significant positive correlation with Ethanol in both sex. The results are in accordance with previous studies. On the other hand, $\gamma$-GTP showed significant positive correlation with Ethanol in males, but not in women. Thus we consider MCV can be used as an indicator of Ethanol in both men and women, while $\gamma$-GTP is an indicator of Ethanol only in men, but not in women. This is a different observation from the previous reports.

We found that MCV in men was related to all drinking factors we considered. $\gamma$-GTP in men was related to Years and strongly to Ethanol. We believe $\gamma$-GTP mainly reflects Ethanol, while MCV reflects not only Ethanol, but also Age, Years, and Days and may indicate drinking habit or even addicts. In women MCV was strongly related to Ethanol, and $\gamma$-GTP was strongly related to Age. Thus we consider $\gamma$ - 
GTP is not a suitable indicator of alcohol intake in women.

MCV increases linearly with aging. ${ }^{[9]}$ One report suggests that increased MCV does not reflect alcohol intake in excessive drinkers 65 years or older. ${ }^{[17]}$ In the present study, MCV increased with aging and this tendency was also apparent in $60 \mathrm{~s}$, although Ethanol decreased from $50 \mathrm{~s}$ to $60 \mathrm{~s} ; 184.4 \mathrm{~g}$ to $167.1 \mathrm{~g}$ in men and $39.0 \mathrm{~g}$ to $26.7 \mathrm{~g}$ in women. This discrepancy suggests that $\mathrm{MCV}$ is not an indicator of drinking in subjects in $60 \mathrm{~s}$, even though they are not excessive drinkers.

One purpose of health check-up is to point out inappropriate life style. A report insists that advice on drinking habit is more effective when based on Ethanol intake than one-day intake. ${ }^{[6]}$ Habitual drinkers often abstain from alcohol several days before health check-up to avoid advice of restricting alcohol. It takes two to four months of abstinence to normalize increased MCV without anemia, caused by drinking. ${ }^{[13], ~[14]}$ In addition, not like $\gamma$-GTP, MCV is not affected by drinking up to 30 days before blood examination. ${ }^{[9]}$ Thus MCV is better than $\gamma$-GTP as a reference when modifying drinking habits.

In Health Japan 21, the recommended alcohol intake is less than or equal to ethanol $22 \mathrm{~g} .{ }^{[10]}$ We estimated the appropriate Ethanol is less than $150 \mathrm{~g}$ for men, and less than $100 \mathrm{~g}$ for women, and determined the cut-off value of MCV and $\gamma$ GTP corresponding to the appropriate Ethanol. The cut-off value of MCV was $92 \mathrm{fL}$ in men, and $90 \mathrm{fL}$ in women. A report claimed that $\mathrm{MCV}$ of more than $94 \mathrm{fL}$ indicates toxic drinking. ${ }^{[2]}$ Our results show that MCV corresponding to the appropriate alcohol intake is still lower. We also found $\gamma$ GTP value of more than $40 \mathrm{IU} / \mathrm{L}$ in men, and $17 \mathrm{IU} / \mathrm{L}$ in women indicates excess alcohol intake even within the reported reference range (0-50 IU/L in men, and 0-30 IU/L in women). ${ }^{[5]}$ We believe MCV is equivalent to $\gamma$-GTP as an indicator of the appropriate alcohol intake.

\section{REFERENCES}

[1] Tatsumi N: Automatic Cell Count. Rinsyou Byouri (Suppl) 97: 284-287,1994 (in Japanese)

[2] Peach HG, Bath NE, Farish S: Predictive Value of MCV for Hazardous Drinkihg in the Community. Clin Lab Haematol
19: 85-87, 1997

[3] Niitsu Y: Iron-deficiency Anemia, Sideroblastic Anemia, Ed. Miwa S, Aoki N, Shibata A, Hematology 2. p. 577-593, Bunkoudou, Tokyo, 1995 (in Japanese)

[4] Abe S: Megaloblastic Anemia, Ed. Miwa S, Hematology 1, p. 523-560, Bunkoudou, Tokyo, 1981 (in Japanese)

[5] Sakai T: Gamma-glutamyl Transpeptidase $(\gamma$-GTP). Nippon Rinsyou 53: 280-283, 1995 (in Japanese)

[6] Mitsumune T, Matsuo K, Mitsumune I, et al.: Usefulness of "Weekly alcohol consumption" as a Guide for Drinkers. Health Evaluation and Promotion 27: 367-371, 2000 (in Japanese)

[7] Ministry of Health, Labour and Welfare, Japan: The National Nutrition Survey in Japan, 2000, p. 107, Daiichisyuppan, Tokyo, 2000 (in Japanese)

[8] Morgan M, Sherlock S: Sex-related Differences Among 100 Patients with Alcoholic Liver Disease. Br Med J 1: 6066, 939-941, 1977

[9] Mundle G, Ackermann K, Munkes J, Mann K: Influence of Age, Alcohol Consumption and Abstinence on the Sensitivity of Carbohydrate-deficient Transferrin, Gamma-glutamyltransferase and Mean Corpuscular Volume. Alcohol 34: 760766, 1999

[10] Health Service Bureau-Ministry of Health and Welfare, Japan: National Health Promotion in the 21st Century "Health Japan 21" (Suppl). Ministry of Health and Welfare, 1999 (in Japanese)

[11] Moore RA: The Diagnosis of Alcoholism in a Psychiatric Hospital. Am J Psychiatry 128: 1565, 1972

[12] Yersin B, Nicolet JF, Dercrey H, Burnier M, van Melle G, Pecoud A: Screening for Excessive Alcohol Drinking. Comparative Value of Carbohydrate-deficient Transferrin, Gamma-glutamyl Transferase, and Mean Corpuscular Volume. Arch Intern Med 155: 1907-1911, 1995

[13] Girard DE, Kumar KL, McAfee JH: Hematologic Effects of Acute and Chronic Alcohol Abuse. Hematol Oncol Clin North Am 1: 321-334, 1987

[14] Chanarin I: Haemopoiesis and Alcohol. Br Med Bull 38: 8186,1982

[15] Whitehead TP, Clarke CA, Whitfield AG: Biochemical and Haematological Markers of Alcohol Intake. Lancet 1: 8071, 978-981, 1978

[16] Clemens MR, Kessler W, Schied HW, Schupmann A, Waller HD: Plasma and Red Cell Lipids in Alcoholics with Macrocytosis. Clin Chim Acta 156: 321-328, 1986

[17] Luttrell S, Watkin V, Livingston G, et al.: Screening for Alcohol Misuse in Older People. Int J Geriatr Psychiatry 12: 1151-1154, 1997 